\title{
Régime par répartition dans l'enseignement supérieur : fondements théoriques et estimations empiriques
}

Contributory Scheme in Higher Education: Theoretical and Empirical

Foundations

David Flacher, Hugo Harari-Kermadec et Léonard Moulin

\section{OpenEdition}

Journals

Édition électronique

URL : http://journals.openedition.org/ei/6233

DOI : 10.4000/ei.6233

ISSN : 2553-1891

Éditeur

Association Économie et Institutions

Référence électronique

David Flacher, Hugo Harari-Kermadec et Léonard Moulin, « Régime par répartition dans

l'enseignement supérieur : fondements théoriques et estimations empiriques », Économie et institutions [En ligne], 27 | 2018, mis en ligne le 20 novembre 2018, consulté le 20 avril 2019. URL

http://journals.openedition.org/ei/6233; DOI : 10.4000/ei.6233

Ce document a été généré automatiquement le 20 avril 2019.

Revue Économie et institutions 


\title{
Régime par répartition dans l'enseignement supérieur : fondements théoriques et estimations empiriques
}

\author{
Contributory Scheme in Higher Education: Theoretical and Empirical \\ Foundations
}

David Flacher, Hugo Harari-Kermadec et Léonard Moulin

\section{Introduction}

1 Les transformations de l'enseignement supérieur depuis la fin des années 1990 ont contribué à développer un vaste marché de l'éducation, au niveau national et international. En Europe, le processus de Bologne a favorisé, avec l'instauration de cycles d'études comparables (Licence, Master et Doctorat) et d'un système européen de transfert et d'accumulation de crédits (ECTS), la mise en place d'un espace européen de l'enseignement supérieur. Parallèlement, au niveau national, des réformes telles que celle associée, en France, à la loi du 10 août 2007 relative aux libertés et responsabilités des universités (dite loi LRU) ont contribué à mettre les universités en concurrence et à les inciter à s'assumer financièrement. Cette logique d'autonomie caractérise les universités de plusieurs pays, notamment anglo-saxons, dont les fonds proviennent désormais en partie des frais d'inscription mais également du mécénat ou d'autres partenariats. Dans une perspective dite du "capital humain", chaque étudiant est amené à investir financièrement dans son éducation, éventuellement en s'endettant, et à rentabiliser cet investissement une fois entré dans la vie active. Ces dimensions caractérisent ce que nous pouvons appeler un « régime par capitalisation » dans l'enseignement supérieur.

2 Est-il possible de définir, par analogie avec le système des retraites, un régime par «répartition» dans l'enseignement supérieur qui serait à la fois plus équitable, plus 
efficient et en mesure de mieux financer le système? Cette question est fondamentale dans la mesure où elle renvoie au débat théorique sur l'efficacité relative d'un système fondé sur le marché, d'une part, et d'un système socialisé reposant sur la solidarité intergénérationnelle et le service public, d'autre part. Elle est fondamentale d'un point de vue politique, car elle renvoie à deux conceptions différentes de l'éducation: l'une comme source de profits futurs et l'autre comme bien collectif. Elle s'inscrit enfin dans une actualité brulante, puisque le relèvement des frais d'inscription a été décidé dans plusieurs pays ou régions du monde et ne cesse de prospérer en France, au-delà des écoles de commerce et de l'enseignement supérieur privé lucratif (Casta 2015) : après Sciences Po (depuis 2004), Dauphine (en 2010), les écoles publiques entrent dans cette dynamique depuis 2014. Ainsi, les Mines de Paris, Centrale-Supélec Télécom ParisTech, Ponts et Chaussées ont, avec d'autres, allègrement dépassé 2000 euros de frais d'inscription (et parfois mêmes 4000 euros pour les étrangers non ressortissants de l'Union européenne)... Ce développement par étapes des frais d'inscription en France n'est probablement pas sans lien avec les vives protestations du monde étudiant et d'une partie des populations concernées dans les pays qui ont réalisé des changements plus brutaux : au Royaume-Uni, des mouvements sociaux de grande ampleur ont secoué le pays à l'autonome 2010 après l'adoption d'une loi autorisant les universités à augmenter leurs frais d'inscription jusqu'à 9000 livres par an; au Québec, où les étudiants se sont fortement mobilisés en 2012 à la suite de l'annonce d'un relèvement progressif des frais d'inscription de 2168 à 3793 dollars canadiens sur la période allant de 2012 à 2017 (soit une augmentation de près de $75 \%$ en cinq ans) aboutissant au retrait du projet et à la chute du gouvernement québécois. De nombreuses manifestations ont également agité le Chili (pourtant soumis à des frais d'inscription élevés depuis l'avènement de la dictature de Pinochet) ou l'Espagne. En France, la vigilance des syndicats concernant les hausses déguisées des frais d'inscription ne semble que retarder un débat inéluctable, les expériences récentes en la matière (Dauphine, Sciences Po, etc.) démontrant l'intérêt des gouvernements pour une tarification des études, y compris à l'université.

Dans cet article, nous résumons les principaux arguments en faveur d'une éducation par capitalisation ainsi que les limites d'un tel régime (section 2). Puis nous définissons théoriquement les principes d'une éducation par répartition et discutons de ses atouts relatifs (section 3). Enfin, nous proposons un chiffrage de ce régime dans le cas français (section 4).

\section{1. Éducation par « capitalisation»}

Dans un article sur la pertinence d'une augmentation des frais d'inscription à l'université, Flacher et al. (2013) définissent ce qu'ils appellent une "éducation par capitalisation ", c'est-à-dire reposant d'abord sur un investissement individuel, matérialisé par le paiement de frais d'inscription et, le cas échéant, par un endettement des étudiants ou de leurs familles. Dans cette perspective, le choix des études et du montant d'investissement associé relève largement d'une décision individuelle, au même titre que relève largement d'une décision individuelle le choix du montant de l'épargne dans un système de retraite par capitalisation. De même, les arguments avancés pour promouvoir un modèle d'éducation par capitalisation ne sont pas sans rappeler ceux avancés en faveur du modèle de retraite par capitalisation (Palier 2003): il s'agit de réduire la dépense socialisée, considérée comme un « poids » par les néolibéraux, au profit d'un transfert de 
la dépense sur l'individu. De ce point de vue, ce sont bien les cotisations (pour les retraites par capitalisation) ou les frais d'inscription (pour l'éducation par capitalisation) qui constituent un investissement préalable dont il s'agit ensuite de tirer un rendement. L'équilibre des "comptes» (entre investissement ou contribution, d'une part, et bénéfices associés, d'autre part) est donc pensé non par année (comme dans le cas de la répartition) mais par génération (comme dans le cas de la capitalisation)1 ${ }^{1}$.

Dans leur article, Flacher et al. (2013) développent une analyse critique de cette forme d'éducation et mettent en avant, pour en démontrer les limites, trois catégories d'arguments mobilisés pour justifier une libéralisation progressive des frais d'inscription à l'université: (i) la quasi-gratuité de l'accès bénéficierait aux classes favorisées, surreprésentées dans l'enseignement supérieur, et serait donc inéquitable, (ii) l'efficience qui serait générée par des mécanismes d'auto-sélection et d'incitation à l'effort, (iii) les besoins de financement de l'enseignement supérieur. Pourtant, comme ils le soulignent, ces arguments rencontrent plusieurs limites importantes.

1. Si l'équité d'un système éducatif peut se définir comme la conjonction d'un principe de compensation (égalité des chances en termes d'accès à un minimum de ressources), d'équité contributive de tous les bénéficiaires de l'enseignement supérieur (définie comme un effort identique de tous les bénéficiaires - étudiants et citoyens profitant des externalités - et donc par progressivité des contributions) et de libertés positives permettant effectivement aux individus des différents milieux sociaux d'aspirer aux mêmes diplômes (égalité des chances effective), alors l'instauration de frais d'inscription n'a presque aucune chance d'être équitable $^{2}$. En effet, il faudrait, pour cela, que le système soit non seulement doté de mécanismes d'aide particulièrement développés et généreux, mais aussi de frais d'inscription progressifs et d'une péréquation des recettes entre établissements situés dans des bassins de recrutement inégalement favorisés. Il s'agit en effet de limiter les mécanismes de polarisation - en termes de ressources et, in fine, en termes sociaux et qualitatifs - au sein de l'enseignement supérieur (Winston 1999; Moulin 2014). Enfin, l'équité contributive appelle aussi à un " partage des coûts » faisant reposer une part significative des coûts sur la fiscalité nationale dans la mesure où l'éducation génère d'importantes externalités positives (Grossman 2006).

2. Si les frais d'inscription étaient relevés, rien ne garantit que le système d'enseignement supérieur gagnerait en efficience : il est d'abord illusoire de croire que chaque individu se comporte « en bon statisticien [calculant] ses chances de gagner tel ou tel salaire demain en fonction des estimations de son talent » (Gary-Bobo \& Trannoy 2005, p. 201) et en agent rationnel. De surcroît, les individus ne disposent souvent ni d'une auto-évaluation juste de leurs talents ni d'une information suffisante sur les universités, les formations et les débouchés professionnels. La rationalité des étudiants est donc fondamentalement limitée en même temps que les choix de parcours apparaissent biaisés par l'origine sociale des étudiants : «le fait d'avoir des chances positives ou négatives d'être, d'avoir ou de faire quelque chose [prédispose], en prédestinant, à agir en sorte que ces chances se réalisent " (Bourdieu 1974, p. 28). Boudon $(1974 ; 1994)$ avait également tiré des conclusions similaires, formalisées depuis dans la théorie de l'aversion relative au risque ${ }^{3}$ selon laquelle la crainte d'un déclassement social serait supérieure au désir d'ascension sociale. L'ambition scolaire dépendrait donc de la position sociale de départ de chaque individu, ce que confirment empiriquement Holm et Jaeger (2008). Ainsi, même lorsqu'existent des prêts à remboursement contingent au revenu $\mathrm{u}^{4}$ (PARC), la perspective, pour certaines populations, de devoir s'endetter conduit à une distorsion des choix de parcours (Cameron \& Heckman 2001 ; Field 2009) défavorable aux milieux populaires. Il en résulterait, d'après Flacher et Harari-Kermadec (2013), un biais d'auto-dévalorisation amenant les candidats à l'enseignement supérieur issus des milieux défavorisés à sous-estimer les rendements privés 
attendus et donc à refuser de payer des frais d'inscription élevés. Enfin, concernant l'efficience, il semble difficile d'établir une relation entre l'effort fourni par les étudiants et le niveau des frais d'inscription (Moulin et al., 2016). Seuls des mécanismes de polarisation peuvent être décrits à travers une polarisation des ressources : les universités les plus riches seraient à même de dispenser un enseignement particulièrement attractif pour les populations les plus talentueuses ou les plus solvables pendant que d'autres universités seraient absorbées dans un cercle vicieux symétrique (voir notamment Winston 1999).

3. L'instauration de frais d'inscription ne permet pas nécessairement d'accroître les moyens financiers des universités. D'abord parce que l'État tend en effet à se désengager du financement de l'enseignement supérieur à mesure que les frais d'inscription prennent le relais ${ }^{5}$. Ensuite parce que les mécanismes d'accompagnement (prêts et/ou bourses) peuvent se révéler particulièrement coûteux pour les finances publiques ${ }^{6}$. S'ajoute à ces coûts un ensemble de dépenses peu productives, en marketing notamment. Enfin, il convient de souligner que les établissements réputés qui, en France, pratiquent des frais d'inscription élevés ne peuvent être considérés comme des modèles généralisables aux universités, et ce même avec le développement du crédit contingent au revenu, dans la mesure où ils sélectionnent leurs étudiants et attirent les plus favorisés d'entre eux.

6 Pour accompagner la hausse des frais d'inscription, des politiques sont souvent mises en place afin de limiter la contrainte d'endettement et de réduire l'aversion au risque qui pourrait éloigner de bons étudiants de l'enseignement supérieur. Par exemple, les PARC peuvent prévoir une annulation de la dette sous certaines conditions. S'ils portent sur des montants trop faibles pour couvrir à la fois les frais d'inscription et le coût de la vie, ils fonctionnent au détriment des classes populaires et moyennes ${ }^{7}$. Ils sont coûteux pour les budgets publics et rendent partiellement caduc les effets d'efficience attendus en accroissant l'anti-sélection et l'aléa moral potentiels (Flacher et al. 2013). Enfin, ils ne favorisent généralement ni l'équité contributive, ni les libertés positives dans la mesure où ils n'effacent que partiellement les effets du poids de la dette. De surcroît, l'objectif de remboursement des prêts est rendu d'autant plus compliqué à atteindre que l'accès à l'enseignement supérieur se massifie et que, malgré des rendements éducatifs élevés, les risques sur le marché du travail s'accroissent (Lochner \& Monge-Naranj 2015).

Enfin, notons que, dans le modèle d'éligibilité de Lévy-Garboua (1976), le modèle d'accumulation du capital humain est lui-même à nuancer dans la mesure où le choix d'études supérieures ne serait pas simplement lié au rendement des études. Selon cette théorie, chaque étudiant maximiserait son utilité, compte tenu de ses contraintes de budget et de temps; et la décision de suivre des études suivrait un double choix : (i) un arbitrage entre le revenu présent et le revenu futur et (ii) un arbitrage entre un accès immédiat à la qualité de vie et un accès futur à l'élite. Ainsi, une réduction des rendements des études pourrait ne pas se traduire par un renoncement aux études mais, dans l'optique de compenser la perte d'utilité future par un gain d'utilité immédiate, par une réduction de l'effort studieux au profit d'un travail rémunéré (au risque de réduire les chances d'obtention d'un diplôme) tout en maintenant constant le temps de loisir pour conserver la qualité de vie de l'étudiant. Ceci serait d'autant plus vrai qu'il n'y aurait ni frais d'inscription ni sélection à l'entrée, comme dans le cas français. Ainsi, en n'éliminant pas totalement ses chances d'obtenir un diplôme, l'étudiant, tout en bénéficiant de son statut, garderait l'opportunité de bénéficier d'un effet de signal associé au diplôme, valorisable sur le marché du travail. Cette théorie, bien que critiquée, s'est révélée relativement cohérente avec les faits, d'après les travaux empiriques de Jarousse (1984) et de Giret (2011). Néanmoins, elle ne permet pas de bien appréhender l'effet du 
développement des formations professionnelles, de l'hétérogénéité des publics et des établissements, ou encore de la nature des compétences acquises par les étudiants. De même, il ne remet pas en cause le cœur de la théorie du capital humain.

8 Face aux limites de la logique de capitalisation que représente un enseignement supérieur fondé sur les frais d'inscription, nous nous proposons de théoriser ce que pourrait être son alternative, le régime d'éducation par répartition.

\section{Fondements théoriques d'un système éducatif par répartition}

\subsection{Définition d'une éducation par répartition}

Nous avons présenté brièvement les principes d'un régime d'éducation par capitalisation dans lequel l'éducation constitue un investissement individuel en capital humain qu'il convient, avant tout, de rentabiliser. Par opposition, nous définissons un régime d'éducation par répartition comme un système considérant avant tout l'éducation comme un bien collectif. Entièrement financé par des prélèvements obligatoires, ce régime consiste à offrir aux étudiants, quelle que soit leur origine sociale, des conditions d'études et de vie leur permettant de mener à bien leur scolarité dans l'enseignement supérieur. L'éducation par répartition doit donc être comprise comme un contrat social fondé sur la solidarité intergénérationnelle et l'équité contributive. Elle permet aux étudiants de réaliser leurs études, le financement de celles-ci reposant sur des prélèvements obligatoires potentiellement progressifs et sur une assiette élargie. La répartition s'inscrit ainsi dans la logique d'un « modèle socialisé » d'éducation.

Du point de vue du financement et des bénéficiaires, la répartition peut s'envisager, pour reprendre l'analogie avec les systèmes d'assurance sociale, dans une perspective que l'on pourrait qualifier de «bismarckienne » : elle est alors financée uniquement par ceux qui ont bénéficié des études, à travers les cotisations sociales de ces derniers. Cette logique n'est pas entièrement déconnectée de la logique du capital humain dans la mesure où $\mathrm{y}$ est implicitement supposé que les études produisent un rendement économique individuel susceptible de financer le système. La répartition pourrait aussi s'envisager dans une logique d'inspiration " beveridgienne " : le financement de l'éducation provient alors d'un impôt dont s'acquittent tous les citoyens, qu'ils aient bénéficié directement de l'éducation ou qu'ils en profitent indirectement à travers les externalités produites. Dans ce second cas, il y a déconnexion complète de la logique d'accumulation du capital humain. Il s'agit alors de privilégier un modèle d'éducation fondé sur l'accès à la connaissance et sur l'émancipation. Une version hybride de la répartition pourrait enfin tenir compte pour partie des rendements individuels (à l'image de l'assurance vieillesse qui inclut en France une composante de répartition - au sens strict - financée par les cotisations sociales et une composante d'aide sociale financée par l'impôt). Notre proposition relève d'une telle hybridation.

11 Ce principe d'éducation par répartition peut être rapproché de celui mis en œuvre dans le cadre de certaines écoles en France. L'exemple le plus emblématique est celui des Écoles Normales Supérieures : les étudiants y sont recrutés et payés en tant que fonctionnairesstagiaires en contrepartie d'un engagement décennal au service de l'État. Il en va de même dans d'autres écoles de formation des fonctionnaires (l'École Nationale 
d'Administration, l'École Nationale des Travaux Publics de l'État, l'École Nationale de la Statistique et de l'Administration Économique, l'École Nationale de la Statistique et de l'Analyse de l'Information, etc.). En cas de non-respect de son engagement décennal, l'étudiant doit rembourser une partie des sommes perçues ${ }^{8}$. Le principe même d'un financement intégral des études (coût de la formation et frais d'entretien) en contrepartie d'un engagement (une contribution financière via les prélèvements obligatoires une fois les études terminées) pourrait s'appliquer à l'ensemble des étudiants dans l'enseignement supérieur. Il relève d'une dimension à la fois bismarckienne (les bénéficiaires contribuent au système par leur engagement) et beveridgienne (à travers un système de financement universel par l'impôt).

De ce point de vue, une éducation par répartition s'oppose « à ce projet de construction d'un marché des étudiants où les droits de scolarité fonctionnent comme des prix, et au financement des études par l'endettement » dénoncé par Vinokur (2009, p. 443).

13 Concrètement, il en résulte qu'une éducation par répartition, de par sa philosophie, doit (i) permettre à tous les étudiants de pourvoir au coût de la vie pendant leurs études, indépendamment de leur milieu social d'origine, grâce à une allocation universelle d'autonomie (section 3.1.1. Montant de l'allocation universelle d'autonomie) financée par des cotisations sociales prélevées sur l'ensemble des actifs et (ii) garantir un financement décent des universités via l'impôt, sans recourir à des frais d'inscription (3.1.2. Refonte du système d'aide et coût net de l'AUA).

\subsubsection{Allocation universelle d'autonomie (AUA)}

14 Dans la suite de cet article, nous entendons par «allocation universelle d'autonomie » (AUA) une allocation accordée sans condition de ressources, à tout étudiant admis dans l'enseignement supérieur afin de lui permettre de mener ses études de manière autonome, c'est-à-dire indépendamment des ressources familiales. Elle est indissociable d'un accès gratuit (ou quasi-gratuit) à l'enseignement supérieur dans la mesure où elle est pensée pour permettre à l'étudiant d'assumer ses dépenses courantes. Elle peut varier selon que l'étudiant est logé par sa famille ou qu'il est décohabitant.

Hypothèse 1. (i) Les coûts de formation ainsi qu'une allocation universelle d'autonomie sont financés par l'impôt et les cotisations sociales. (ii) Le montant de l'AUA ne dépend que du besoin de l'étudiant de se loger'.

16 Nous développons dans la suite de l'article les mesures d'accompagnement de l'AUA qui nous semblent nécessaires pour assurer l'équité (2.3) et l'efficience (2.4) du financement de l'enseignement supérieur, et ce notamment afin de lutter contre l'anti-sélection et l'aléa moral.

\subsubsection{Accroissement des moyens des universités}

17 La France se caractérise par un dualisme très marqué du financement de son enseignement supérieur : en 2015-2016, le coût annuel moyen du système universitaire par étudiant était de 10387 euros à l'université contre 15100 euros en classes préparatoires aux grandes écoles (CPGE) ${ }^{10}$. Les différences de dépenses publiques par étudiant en fonction du diplôme sont encore plus impressionnantes puisque, selon Courtioux (2009), les dépenses publiques s'élèvent à 37294 euros, en moyenne, pour un 
diplôme de niveau BAC +5 , s'échelonnant de 17805 euros pour un étudiant de master à 127527 euros pour un diplômé d'une « très grande école d'ingénieur ${ }^{11}$ ».

18 Ces disparités bénéficient aux populations socialement les plus favorisées au détriment des autres : les élèves qui ont le plus de moyens vont dans des établissements prestigieux, dans lesquels les dépenses de l'État par étudiant sont plus importantes, tandis que les élèves issus des classes sociales les moins aisées, quand ils font des études, se retrouvent dans des filières pour lesquelles la dépense étatique est moindre. Ainsi, l'effectif des CPGE était composé, en 2016-2017, à moitié d'enfants de cadres et de professions intellectuelles supérieures $(51,7 \%)$ contre seulement $7,2 \%$ d'enfants d'ouvriers, tandis que celui de l'université était composé à $34,1 \%$ d'enfants de cadres et à $12 \%$ d'enfants d'ouvriers (RERS 2017). Selon Trannoy (2006, p. 747) : «il serait [donc] pour le moins paradoxal de ne pas aligner les moyens des établissements accueillant aujourd'hui les élèves de milieux défavorisés sur ceux des classes préparatoires et des grandes écoles ». C'est la raison pour laquelle nos hypothèses incluent une augmentation de 5000 euros de dépenses par étudiant en licence à l'université. Ces dépenses permettront en effet d'améliorer à la fois l'encadrement et les conditions de travail à l'université mais également de mettre en place des mécanismes d'accompagnement des étudiants en difficultés.

19 Ce renforcement des moyens affectés à l'université française apparaît d'autant plus prégnant qu'on les compare aux niveaux de financement de l'enseignement supérieur à l'étranger. C'est ainsi qu'en s'appuyant sur les travaux du CERC (2003) ou de l'OCDE, Trannoy (2006, p. 747) affirme que «l'université française [...] est sous-financée par rapport à ses concurrents français ou étrangers ». Ainsi, en 2014, la France consacrait $1,5 \%$ de son PIB à l'enseignement supérieur (dont $1,2 \%$ provenant des dépenses publiques) contre $2,7 \%$ du PIB (dont $0,9 \%$ provenant des dépenses publiques) aux ÉtatsUnis et 2,6\% du PIB (dont 1,3\% provenant des dépenses publiques) au Canada (OCDE, 2017). La moyenne des pays de l'OCDE s'établissant à $1,6 \%$ du PIB (dont 1,1\% provenant des dépenses publiques). Ces différences traduisent des écarts considérables en matière de dépenses par étudiant. En 2014 toujours, celles-ci étaient en moyenne de 16422 USD en France, contre 29328 USD aux États-Unis, 21326 USD au Canada et 16143 USD en moyenne pour les pays de l'OCDE ${ }^{12}$.

Ces chiffres sont évidemment à prendre avec précaution: il s'agit de moyennes qui cachent parfois d'importantes disparités intra-nationales et qui dépendent non seulement des modes de calcul, mais aussi des filières éducatives, du choix d'orientation des étudiants, des choix pédagogiques, du niveau des salaires des enseignants dans le pays considéré, etc. De surcroît, ils ne nous renseignent pas sur le caractère « optimal » d'un système ou d'un niveau de dépense. Néanmoins, ces chiffres nous permettent de souligner l'écart entre les dépenses françaises pour l'enseignement supérieur et celles des pays de l'OCDE les plus avancés, tendant à accréditer l'hypothèse d'un sousinvestissement en France. augmenter de 5000 euros. 


\subsection{L'éducation par répartition comme extension de la sphère publique}

\subsubsection{D'un financement direct du capital humain (par les frais d'inscription et l'endettement) à un financement indirect de l'éducation par la solidarité intergénérationnelle}

Maurin (2007) s'appuie sur une analogie avec le système de retraite par répartition pour proposer un mode de financement de l'enseignement supérieur. Cependant, ses propositions s'inscrivent dans une vision individuelle de la participation, sous la forme de frais d'inscription et de PARC: la contribution est liée au bénéfice individuel tiré de l'enseignement supérieur. Cette approche élude donc une dimension du système de répartition, à savoir la solidarité intergénérationnelle. Or dans notre modèle, ce sont bien les actifs d'aujourd'hui qui payent pour les futurs actifs. De même que le système de retraite par répartition lie les actifs, en aval, aux anciens actifs, les retraités, l'éducation par répartition les lie, en amont, aux futurs actifs que sont les étudiants. Cette solidarité intergénérationnelle contribuerait ainsi à placer les actifs au centre d'un système de double identification : non seulement au retraité qu'il deviendra mais aussi à l'étudiant qu'il a été. Dans cette logique, comme dans le cas des retraites par répartition, «les comptes sont équilibrés par année civile : ce que la caisse touche une année donnée est distribué la même année » (Bourgeois-Pichat 1978, p. 1115) ${ }^{13}$.

De plus, financée par des cotisations sociales (potentiellement progressives), l'AUA tendrait à réduire les écarts entre les rendements des formations et à valoriser les critères non pécuniaires : tout en restant en partie guidés par les besoins sur le marché du travail, les comportements apparaîtront plus libres car davantage guidés par les préférences individuelles, les débouchés attendus et la valeur symbolique du diplôme. Autrement dit, l'allocation universelle d'autonomie et l'absence de frais ne suppriment pas les incitations à répondre aux attentes du marché mais les relativisent. La question de l'efficacité économique, mais aussi sociale, du système peut dès lors se déplacer vers le débat politique et démocratique sur le service public de l'éducation, incluant la question du nombre de places à offrir dans les différentes filières et les conditions d'accès à ces filières.

L'AUA et l'accroissement du financement des universités, financés par des prélèvements obligatoires, participent donc d'une logique opposée à celle de la théorie du capital humain : l'éducation constitue d'abord un investissement collectif, dont les retombées sont aussi (ou avant tout) sociales. Ces externalités ne sont pas seulement liées au fait que les diplômés, de par leurs revenus plus élevés, payent davantage d'impôts ${ }^{14}:$ l'éducation permet un partage des savoirs, contribue au développement des consciences et à l'émancipation de chacun.

Comme pour les retraites des régimes par répartition, ce mécanisme de solidarité présente de nombreux avantages : il est peu sensible aux conjonctures économiques et repose sur un financement pérenne et potentiellement équitable. Il prémunit l'éducation d'une financiarisation, c'est-à-dire d'un recours croissant à l'endettement et aux marchés financiers, qu'ont pu connaître certains secteurs économiques et dont les analyses récentes ont montré les risques sous-jacents (Belfield 2017). De plus, il est susceptible de contrebalancer les effets, parfois négatifs, des évolutions démographiques: un 
vieillissement de la population accroît les besoins de financement du système de retraite, mais réduit ceux du régime d'éducation par répartition, et inversement (pour peu que les retraités soient mis, dans une certaine mesure au moins, à contribution du financement de l'éducation par répartition). En mutualisant les risques, il encourage les populations les moins favorisées à entreprendre des études, alors même que leur milieu social d'origine ne les y prédisposait pas. Il donne une possibilité réelle, pour ces étudiants, d'envisager des études longues ou plus prestigieuses, ce qui reste impossible pour ceux qui sont contraints aujourd'hui d'exercer une activité rémunérée pour financer leurs études. Il offre une indépendance à l'étudiant et soulage les familles modestes.

Les deux modes de financement diffèrent donc sensiblement dans la mesure où la capitalisation confie l'arbitrage en matière d'investissement éducatif aux choix individuels ${ }^{15}$ et les soumet aux aléas conjoncturels, alors que la répartition pose explicitement cet arbitrage dans un cadre politique: l'offre éducative et l'allocation relèvent de décisions collectives dont nous montrons, dans la suite de cet article, qu'elles peuvent se révéler à fois les plus efficaces et les plus équitables (cf. sections 2.4 et 2.3).

\subsubsection{Service public contre service marchand de l'éducation : une typologie des systèmes éducatifs}

27 L'opposition entre ce que nous appelons une éducation par capitalisation et une éducation par répartition peut-être rapprochée de celle développée par Vinokur (2007) entre un « modèle marchand » d'éducation et un « modèle socialisé » (tableau 1).

Tableau 1. Modèles d'éducation socialisé et marchand

\begin{tabular}{|c|c|c|}
\hline & Modèle marchand & Modèle socialisé \\
\hline Nature du bien éducatif & Bien privé & Bien public \\
\hline $\begin{array}{l}\text { Caractère marchand ou } \\
\text { non }\end{array}$ & $\begin{array}{l}\text { Service non } \\
\text { marchand }\end{array}$ & Service marchand \\
\hline Nature lucrative & $\begin{array}{l}\text { Lucratif ou non } \\
\text { lucratif selon le cas }\end{array}$ & Non lucratif \\
\hline Tarif/prix & Payant & Gratuit ou quasi gratuit \\
\hline offre & $\begin{array}{l}\text { Déterminée par le } \\
\text { marché }\end{array}$ & $\begin{array}{l}\text { Déterminée (démocratiquement) par les } \\
\text { besoins économiques et sociaux }\end{array}$ \\
\hline Sélection & $\begin{array}{l}\text { Académique et/ou } \\
\text { financière }\end{array}$ & Académique \\
\hline $\begin{array}{l}\text { Financement du service } \\
\text { d'enseignement }\end{array}$ & Privé & Public \\
\hline $\begin{array}{l}\text { Prise en charge des } \\
\text { dépenses d'entretien }\end{array}$ & $\begin{array}{l}\text { Par la famille ou par } \\
\text { l'emprunt }\end{array}$ & Salaire/bourse/allocation d'autonomie \\
\hline
\end{tabular}




\begin{tabular}{|l|l|l|}
\hline Accès à l'emploi & Marché du travail & $\begin{array}{l}\text { Affectations administrées, salaires définis } \\
\text { par les barèmes nationaux (visant l'égalité) }\end{array}$ \\
\hline
\end{tabular}

Source : inspiré de Vinokur (2007)

Le modèle marchand se caractérise avant tout par l'instauration du principe de tarification des études, d'un accès à l'enseignement supérieur par l'endettement et d'une concurrence entre établissements (avec les mécanismes de polarisation et de ségrégation possiblement induits, Flacher et al. 2013). Tandis que le modèle socialisé présente les caractéristiques d'un service public et notamment l'égalité de traitement des usagers (où qu'ils se trouvent sur le territoire et indépendamment de leurs conditions sociales), la neutralité et la transparence. Le modèle marchand s'est largement développé et continue de se développer depuis trente ans avec le soutien de l'OCDE : les États-Unis, le RoyaumeUni, l'Australie, le Canada, le Chili sont autant d'exemples de pays particulièrement avancés en la matière. Les systèmes s'approchant d'une logique d'éducation par répartition ne sont, en revanche, pas légion. De nombreux pays se trouvent dans une position intermédiaire (à commencer par la France, où les frais d'inscription sont faibles mais les moyens des universités et les allocations pour les étudiants particulièrement limités). Dans ce paysage, les pays du nord de l'Europe (Danemark, Finlande, Norvège et Suède) font figure d'exceptions en se rapprochant le plus d'un système d'éducation par répartition : dans ces pays, aucun frais d'inscription n'est demandé et, en plus de bourses (ou d'allocations dans le cas du Danemark et de la Suède), un accès à des prêts aménagés est ouvert à tous. Ces prêts permettent de couvrir les frais d'entretien, leurs taux sont subventionnés et la dette peut parfois être effacée pour certaines études, métiers ou régions d'installation. Comme le note Levy (2004), la conjonction de l'absence de frais d'inscription et de prêts aménagés relève d'une politique publique de répartition intergénérationnelle des revenus, avec pour objectif de limiter les écarts de revenus :

[...] la fraction de la génération active qui a bénéficié des prêts assure par ses remboursements une partie du financement de l'éducation de la génération suivante; le reste, abondé par la fiscalité progressive qui pèse sur l'ensemble des revenus de la génération active, représente la couverture collective des frais de socialisation des jeunes, i. e. des externalités. Cet exemple montre qu'un système de prêts aux étudiants peut, dans un contexte politique favorable, participer d'un partage des coûts dont le but n'est pas de réduire la part du financement public mais d'assurer en longue période, et de manière transparente, un double objectif politique de répartition intergénérationnelle des moyens d'existence et de sécurisation de la reproduction des capacités (Vinokur 2007, p. 213).

Néanmoins, si les systèmes nordiques revêtent quelques caractéristiques d'une éducation par répartition, le poids de la dette, même limité, demeure un problème pour les étudiants les moins favorisés en même temps qu'il est source d'inégalités. Enfin, même limité et géré par une institution publique, le mécanisme d'endettement répond à une logique d'individualisation plus caractéristique de la capitalisation que de la répartition, pouvant conduire à une forme de "docilisation" de la population étudiante et à une division de la force politique que le monde étudiant est susceptible de représenter ${ }^{16}$.

Fondée sur une péréquation des moyens entre établissements au niveau national et sur des aides identiques pour tous, l'éducation par répartition permet à chaque étudiant, où qu'il soit sur le territoire et quelle que soit son origine sociale, d'entreprendre les études de son choix. Elle respecte ainsi le principe d'égalité de traitement alors que l'éducation 
par capitalisation tend à polariser le système d'enseignement supérieur et à privilégier les ménages et territoires les plus favorisés (cf. Flacher et al. 2013). La répartition appellerait un débat transparent sur l'allocation des moyens et des aides aux étudiants (permettant d'adapter le service public aux besoins) ainsi que sur les barèmes des prélèvements obligatoires (permettant de faire contribuer chacun en fonction de ses capacités contributives).

31 Peut-on dès lors penser une éducation par répartition comme un système socialisé efficace, c'est-à-dire à la fois (i) équitable, (ii) capable de dégager les financements nécessaires et (iii) efficient du point de vue allocatif et productif.

\section{3. Équité et capacité de financement de l'enseignement supérieur dans le cadre d'une éducation par répartition}

32 L'éducation par répartition, telle que nous l'avons définie constitue d'abord un important vecteur d'équité : elle établit des mécanismes de compensation (i) en finançant mieux les filières jusque-là sous financées et dans lesquelles les étudiants des milieux modestes sont surreprésentés; (ii) en soulageant les familles (au premier rang desquelles les plus modestes) et en accordant, grâce à l'AUA, une autonomie comparable pour tous les étudiants (quel que soit leur milieu social d'origine). En rendant moins nécessaires les transferts intergénérationnels au sein des familles, de même que l'exercice d'activités rémunérées ${ }^{17}$, l'AUA s'attaque à la reproduction des inégalités.

L'éducation par répartition, si elle repose sur des prélèvements obligatoires et sur une assiette suffisamment large, peut également contribuer à l'équité contributive du système éducatif. Comme nous l'avons souligné, elle peut être considérée de manière complètement déconnectée de la logique d'accumulation du capital humain, en étant financée par l'ensemble des contribuables. Mais elle peut aussi garder un lien avec l'accumulation du capital humain en intégrant une contribution progressive spécifique portant sur les anciens étudiants. Ainsi, pour Glennerster et al. (1968, p. 26), un « impôt progressif permettrait à la communauté de recouvrer la valeur des ressources dédiées à l'enseignement supérieur en s'appuyant sur ceux qui en ont personnellement tiré bénéfice ». Il permettrait, de surcroît, de lever davantage de fonds publics qu'un système de frais d'inscription avec PARC (Glennerster et al. 2003). Cependant, si Glennerster et al. (1968; 2003) envisagent une fiscalité sur les seuls diplômés du supérieur, l'équité commande d'envisager une assiette plus large, tenant compte non seulement des rendements individuels mais aussi des rendements sociaux de l'éducation. Tout en offrant la même allocation à tous les étudiants, l'impôt doit donc s'appuyer sur un barème "juste», c'est-à-dire un barème qui redistribue les revenus selon les capacités contributives de chacun des anciens étudiants (capacités essentiellement liées à leur qualification) et des citoyens (qui profitent du niveau général d'éducation). De ce point de vue, la fiscalisation des rendements privés de l'éducation n'apparaît pas éloignée du principe des $\mathrm{PARC}^{18}$. Une fiscalité nationale, plutôt que locale, apparaît alors préférable pour bénéficier d'une information fine sur les revenus (inaccessible aux établissements d'enseignement supérieur s'ils devaient réaliser le recouvrement), pour mutualiser les moyens de collecte de l'impôt et pour favoriser une mutualisation des fonds réunis ${ }^{19}$. Financée de manière progressive, l'éducation par répartition permettra de réduire les rendements privés associés à de nombreuses écoles prélevant des frais d'inscription élevés, avec pour conséquence d'en limiter la hausse éventuelle voire d'en provoquer la 
baisse $^{20}$, contribuant là aussi à privilégier (sur les calculs de rendements) l'adéquation entre les choix d'études, les talents et les préférences individuels.

Enfin, l'éducation par répartition, et notamment l'AUA, peuvent contribuer à accroître les libertés positives. L'AUA supprime le poids de la dette et ses effets ségrégatifs. Elle pourrait, également, sans stigmatiser une population, contribuer à rendre les cursus du supérieur attractifs pour des étudiants et des familles qui, jusque-là, peinaient à appréhender et considérer la perspective d'études coûteuses. C'est notamment le cas des filières les plus sélectives et les plus prestigieuses, dont la durée et la densité des enseignements ne sont pas compatibles avec l'exercice d'une activité rémunérée. Par ailleurs, il convient de penser des mécanismes permettant de pallier les difficultés d'orientation que connaissent les étudiants issus des classes sociales les moins favorisées, comme l'accord d'un "droit à l'erreur» ou la mise en place de mécanismes de réorientation ${ }^{21}$. De ce point de vue, l'AUA apparaît complémentaire d'un meilleur financement public des universités: l'AUA permet d'émanciper les étudiants d'une contrainte financière, pour leur permettre d'accéder à l'enseignement supérieur et de se consacrer pleinement à leurs études ; tandis que l'augmentation des dépenses en premier cycle universitaire vise précisément à donner les moyens financiers aux universités de faire réussir leurs étudiants. Les effets de l'université d'appartenance sur la réussite aux études et les choix de spécialisation (voir par exemple Nicourd et al. 2012) s'en trouveront diminués puisque, d'une part, l'augmentation des moyens accordés aux universités vise également à réduire les disparités existantes au niveau territorial entre établissements d'enseignement supérieur et puisque, d'autre part, l'AUA sera favorable à la décohabitation des étudiants vivant dans une zone où le maillage universitaire est aujourd'hui insuffisant. Enfin, l'augmentation des moyens accordés aux universités pourra permettre un rééquilibrage des moyens accordés aux différentes filières de l'enseignement supérieur. Notre proposition apparaît dès lors à la fois plus juste et plus efficiente car, au-delà du financement, elle tend à intégrer l'ensemble des étudiants potentiels, qu'ils soient issus des milieux modestes ou favorisés.

Il ne faut néanmoins pas considérer que l'AUA peut, à elle seule, résoudre les nombreux problèmes qui réduisent les chances de voir un individu issu des milieux populaires se saisir efficacement de l'opportunité que représente cette allocation. D'importantes difficultés demeurent également en amont, dans le système éducatif. Elles dépassent néanmoins très largement le cadre de cet article.

Nous déduisons des éléments qui précèdent la proposition suivante.

Proposition 1. Pour garantir une relative équité d'accès à l'enseignement supérieur, l'éducation par répartition doit (i) reposer sur un système national de prélèvements obligatoires, progressif et étendu aux non-résidents ayant bénéficié d'études supérieures au niveau national, (ii) portant sur l'ensemble des actifs - voire des retraités - (pour les cotisations sociales finançant l'AUA) et des citoyens (pour les impôts finançant les dotations aux établissements) dans la mesure où l'éducation bénéficie à l'ensemble de la communauté nationale, (iii) prévoir un accompagnement des étudiants dans leurs choix d'orientation qui reconnaisse les talents issus des différentes classes sociales (années propédeutique et formes d'examens et de concours adaptés).

\subsection{Efficience d'un système d'éducation par répartition}

Un régime d'éducation par répartition se caractérise par une stabilité vis-à-vis des aléas conjoncturels, l'absence de financiarisation et la réduction de la dépendance aux comptes 
publics. À quelles conditions est-il également efficient $\mathrm{du}$ point de vue microéconomique? Indirectement, une éducation par répartition peut avoir un pouvoir incitatif sur les universités accueillant des étudiants entièrement consacrés à leurs études. Qu'en est-il des incitations qu'ont les étudiants à les mener à bien? Pour inciter les étudiants à l'efficience dans le cadre d'un régime d'éducation par répartition, des mécanismes doivent limiter les opportunismes liés à l'attribution de l'AUA, qu'il s'agisse d'anti-sélection ou d'aléa moral, tout en offrant aux étudiants un "droit à l'erreur ». Enfin, il faut que le système, tout en veillant à une bonne utilisation des fonds, garantisse un financement récurrent évitant une dispendieuse concurrence.

\subsubsection{Lutter contre l'anti-sélection}

L'anti-sélection peut advenir (i) lorsque des étudiants souhaitent bénéficier de l'AUA en s'engageant dans une filière pour laquelle ils ne sont objectivement pas qualifiés ou dans une filière n'offrant pas (ou peu) de débouchés. Elle peut aussi se manifester (ii) chez des étudiants qui, parce que particulièrement doués et/ou dotés, préfèreraient ne pas participer au contrat social d'une éducation par répartition (autrement dit ne pas recevoir l'AUA et ne pas la financer en tant que contribuable).

Pour éviter le comportement de ces derniers, l'AUA ne doit pouvoir être refusée par aucun étudiant. Pour pallier le risque d'anti-sélection lié à la potentielle inadéquation du profil des étudiants à la filière choisie, le système d'enseignement supérieur doit :

(i) interdire (sauf exception), le cumul de revenus avec l'AUA. L'AUA doit en effet permettre à l'étudiant de se consacrer pleinement à ses études et ne doit pas être considérée comme un complément de revenu d'une autre activité.

(ii) Le système d'enseignement supérieur doit également fournir l'information la plus complète, claire et utile possible aux étudiants sur les débouchés de leurs études. Rendre possible la simple observation (par les étudiants) des conditions objectives d'accès au marché du travail à l'issue d'un cursus peut en effet, en objectivant les conséquences d'une décision, contribuer à lutter contre cette anti-sélection.

43 (iii) L'État doit enfin décourager les choix qui seraient objectivement peu pertinents (sans pour autant avoir recours aux frais d'inscription) et éviter d'admettre des étudiants qui, même aidés, ne parviendraient pas à réussir les études qu'ils souhaitent entreprendre. Pour autant, il faut se garder d'introduire des formes de sélection qui introduiraient un biais défavorable aux classes sociales les moins favorisées (Flacher \& Harari-Kermadec 2013). Il faut penser la nature même des mécanismes d'orientation afin de corriger les discriminations qu'ils induisent. Plutôt que de refermer en amont l'accès à l'université, nous proposons que la prorogation de l'AUA, au-delà de l'année en cours, soit strictement soumise à la réussite de l'étudiant et le « droit à l'erreur » encadré.

Concrètement, nous suggérons la mise en place d'une « commission d'orientation » ayant vocation à évaluer la pertinence a priori des choix d'orientation ${ }^{22}$. Cette commission agirait en aval des conseillers d'orientation. Elle contribuerait, par les incitations qu'elle produit, à limiter les situations d'échec et à réduire le coût lié à une longue et inutile stagnation de certains étudiants dans des filières qui ne leur correspondent pas. Elle pourrait se composer d'universitaires (accompagnés d'enseignants du secondaire pour les inscriptions en licence 1), d'usagers (les étudiants bénéficiaires de l'AUA) et de représentants des partenaires sociaux, sous tutelle de l'État, comme pour les autres branches de la sécurité sociale ${ }^{23}$. Alimentée par les avis motivés des équipes enseignantes 
qui ont eu la charge de l'étudiant, la commission donnerait ainsi un avis (favorable ou réservé à l'entrée dans une filière), accompagné d'éventuels conseils, sans pour autant imposer une orientation aux étudiants ${ }^{24}$. Le non-respect de cet avis aurait alors pour conséquence, en cas d'échec de l'étudiant, la non-prorogation de son droit à l'AUA. Inversement, l'étudiant pourrait, sous certaines conditions, bénéficier d'un «droit à l'erreur » dans le cas où cet avis aurait été respecté.

\subsubsection{Réduire l'aléa moral tout en accordant un « droit à l'erreur »}

Si le versement de l'allocation universelle d'autonomie est déclenché par l'admission dans un établissement d'enseignement supérieur, sans condition de ressources, il apparaît indispensable de limiter l'aléa moral (cas des étudiants ne fournissant pas les efforts nécessaires à la réussite de leur cursus). Un étudiant pourrait s'inscrire sur une longue période sans pour autant progresser dans son cursus. Conditionner l'AUA à la réussite des examens, à l'assiduité et au non-cumul de revenus, inciterait les étudiants à achever leur cursus dans un délai raisonnable. Ainsi, par exemple, un (seul) redoublement par niveau d'étude pourrait être autorisé avec maintien de l'AUA, sous réserve que l'étudiant ait fait preuve de sa motivation et de ses efforts en ayant validé au moins la moitié des crédits de l'année concernée et l'ensemble des modules des années antérieures. Il s'agit ainsi de s'assurer que l'étudiant fournit un travail régulier et significatif et qu'il a bien le niveau requis pour poursuivre des études dans la voie qu'il a choisie. En revanche, dans le cas de résultats trop faibles, d'une motivation manifestement dégradée et en l'absence de réorientation, la suspension de l'AUA prendrait effet jusqu'à la réussite d'au moins la moitié des crédits de l'année concernée. De même, l'AUA serait suspendue en cas d'échec, si l'étudiant a choisi une filière ouvertement contraire aux recommandations a priori de la commission d'orientation.

Néanmoins, si conditionner l'AUA à la réussite aux examens apparaît essentiel, les conditions de suspension de l'allocation doivent tenir compte d'un droit raisonnable à l'erreur, que cette erreur concerne le choix d'orientation des étudiants ou les difficultés d'adaptation au monde universitaire. Cet aspect est d'autant plus essentiel que les difficultés d'orientation ou d'adaptation sont plus importantes dans les classes sociales les moins favorisées, pour lesquelles les cursus et débouchés sont souvent moins bien connus. Il renvoie plus généralement au problème d'équité du système éducatif : un étudiant issu d'un milieu modeste et craignant de ne pas réussir dans une filière pourrait choisir d'être moins ambitieux dans le choix de sa formation pour limiter les risques de perdre le bénéfice de l'AUA. Il convient donc de mettre en place des mécanismes pour contrebalancer cet effet d'anti-sélection. L'AUA pourrait notamment être prorogée sous certaines conditions. Cette prorogation pourrait dépendre conjointement des conseils d'orientation prodigués à l'étudiant en amont du choix de sa formation, des efforts fournis et de la régularité de son travail. Elle pourrait être de droit en cas de réorientation précoce ${ }^{25}$ et contingente à la décision de la commission en cas de réorientation plus tardive.

Enfin, pour lutter contre le découragement d'étudiants inscrits dans une filière qui ne leur correspond pas ou d'étudiants en difficulté, la hausse des moyens accordés aux universités dans le cadre d'une éducation par répartition doit permettre (i) la mise en place d'années de transition (visant à fournir les prérequis aux étudiants souhaitant démarrer une formation pour laquelle ils n'étaient pas préparés) et (ii) la mise en place de 
classes de rattrapage (susceptibles de permettre aux étudiants en difficulté de rebondir l'année suivante).

48 L'ensemble des conditions d'efficience de l'AUA peuvent donc être résumées de la manière suivante :

Proposition 2. (i) Chaque étudiant - résidant ou non dans le pays ${ }^{26}$ - est tenu d'accepter l'AUA ainsi que l'engagement correspondant, une fois actif, de participer au financement de l'enseignement supérieur (et ce, indépendamment de son pays de résidence ${ }^{27}$ ). (ii) l'AUA ne doit pas être cumulable avec d'autres revenus (sauf exception décidée par une commission ad hoc) et doit pouvoir être suspendue si les conditions d'assiduité ne sont pas remplies. (iii) Les étudiants doivent être informés le plus complètement et utilement possible sur les débouchés des filières de l'enseignement supérieur. (iv) L'université devra pouvoir conditionner la prorogation de l'AUA audelà de l'année en cours à la réussite (au moins partielle) des examens de l'année en cours, à la pertinence $d u$ choix de la filière et $d u$ parcours ou à une réorientation. (v) Une commission d'orientation pourrait être créée pour donner un avis sur les choix d'orientation (ou de réorientation), avis sur lequel pourrait s'appuyer une suspension de l'AUA en cas d'échec. Elle pourrait également décider de la prorogation de l'AUA, sous certaines conditions. (vi) Pour réduire les biais de sélection entre classes sociales et favoriser ainsi une massification efficiente des études supérieures, pourront être mis en place des années de transition, des classes de rattrapage et des mécanismes d'accompagnement de l'orientation des étudiants adaptés à la diversité des cultures et des origines sociales.

\subsubsection{Réduire la concurrence et les phénomènes de polarisation pour davantage d'efficience productive}

Comme nous l'avons souligné dans la section 2, lorsque les frais d'inscription constituent un enjeu financier pour les établissements, ceux-ci se livrent à une concurrence dispendieuse du fait des frais de communication ${ }^{28}$ et de confort, de même qu'à des politiques produisant inflation et inégalités salariales au sein des universités (Espeland \& Sauder 2007). Ces dépenses sont contestables en termes d'efficacité pédagogique comme en termes d'efficience productive et participent de la polarisation entre établissements ${ }^{29}$. Elles détournent les universités de leurs missions et leur budget de leurs objectifs premiers.

51 Une solution réside donc dans une réduction de cette concurrence dispendieuse et par un accroissement des moyens mis à disposition des universités, ces moyens constituant en effet une condition nécessaire pour permettre aux universités de se concentrer sur leurs missions et de mieux les assurer. Dans cette logique, les universités situées sur des bassins de recrutement socialement peu favorisés devraient être dotées de financements spécifiques pour assurer ces missions. Des aménagements pourraient ainsi être envisagés pour les étudiants suffisamment doués et motivés mais dont les lacunes justifient une prise en charge particulière (soutien, tutorat, classe à effectif réduit, année propédeutique, etc.).

Un contrôle a posteriori des dépenses peut, pour sa part, prévenir d'éventuelles dérives. 


\section{Financement d'une éducation par répartition : essai de chiffrage}

\subsection{Allocation universelle d'autonomie : chiffrage et pistes de financement}

\subsubsection{Montant de l'allocation universelle d'autonomie} déduisons alors, en prenant comme référence le nombre d'étudiants de l'année 2015-2016

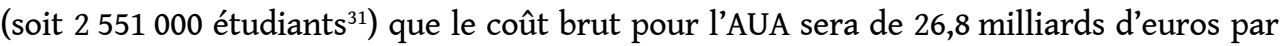
an.

L'AUA étant destinée à couvrir les coûts de la vie relatifs à la poursuite d'étude, nous proposons que son montant dépende du mode d'hébergement de l'étudiant : un étudiant hébergé chez ses parents percevra moins qu'un étudiant qui ne l'est pas, dans la mesure où celui-ci doit généralement s'acquitter d'un loyer.

D'après l'enquête « conditions de vie » 2016 réalisée par l'Observatoire de la Vie Étudiante (OVE), les dépenses mensuelles moyennes des étudiants décohabitants s'élèvent à 765 euros (loyer compris) et celles des étudiants vivant chez leurs parents à 342 euros. Selon l'OVE (2017b), le loyer moyen d'un étudiant est de 430 euros, celui-ci étant plus élevé en région parisienne (560 euros dans la petite couronne et 543 euros dans la grande couronne) et à Paris (637 euros).

En établissant le montant de l'allocation universelle d'autonomie, hors loyer, à 600 euros (sur 12 mois), les étudiants pourraient donc se rendre entièrement disponibles pour leurs études et les mener dans de bonnes conditions. Nous proposons ensuite de compléter l'AUA par 400 euros pour les étudiants qui ne vivent plus chez leurs parents et qui ont un logement à charge. Ce complément pourrait être modulé suivant le lieu d'habitation sur le modèle des aides personnalisées au logement (APL), mais la prise en compte d'un niveau unique suffit dans la perspective du chiffrage national retenue dans cet article.

Ainsi le montant total de l'AUA pourrait s'élever à 600 euros par mois (7 200 euros par an) pour les étudiants vivant encore chez leurs parents et à 1000 euros par mois (12 000 euros par an) pour les étudiants décohabitants. Ces sommes sont comparables à celles attribuées en Suède et au Danemark ${ }^{30}$ et nettement supérieures à celles avancées par Gary-Bobo \& Trannoy (2005) (670 euros/mois).

Parmi l'ensemble des étudiants, 31,3\% vivent au domicile parental (OVE, 2017b). Nous en

Nos calculs ne tiennent pas compte de l'augmentation du nombre d'étudiants qui pourrait résulter de l'instauration de cette mesure. En effet, bien qu'une augmentation du nombre d'étudiants inscrits soit probable, l'AUA vise également à réduire l'échec en premier cycle (notamment en faisant en sorte que les étudiants n'aient pas besoin de travailler pour financer leurs études et en définissant des conditions de réussite à son maintien). On peut donc raisonnablement supposer que le taux de redoublement sera sensiblement réduit non seulement grâce à l'AUA, mais également grâce à l'augmentation des moyens des établissements en licence. De surcroit, la mise en place de l'allocation d'autonomie permettrait, à terme, de réaliser des économies sur l'indemnisation du chômage (l'AUA excluant une activité professionnelle rémunérée parallèle aux études). À défaut de pouvoir quantifier précisément les évolutions contraires du nombre d'étudiants et des 
indemnisations annulées du chômage, nous faisons l'hypothèse que le nombre d'inscrits n'évoluera pas significativement avec la mise en œuvre de l'AUA ${ }^{32}$. Enfin, même si le système que nous proposons conduisait à un accroissement de la demande d'éducation, les talents ainsi formés produiraient aussi davantage de richesses dans le pays et donc de ressources disponibles pour financer l'enseignement supérieur. Néanmoins, la logique première de notre proposition dépasse ces considérations: nous pensons l'allocation universelle d'autonomie comme le moyen d'exercer pleinement un droit aux études supérieures et non comme une incitation économique visant indirectement un accroissement du PIB. Nous ne traitons pas ici des effets macroéconomiques que pourrait entraîner la mise en place d'un financement de l'enseignement supérieur par répartition.

\subsubsection{Refonte du système d'aide et coût net de l'AUA}

La mise en place de l'AUA doit s'accompagner d'une refonte du système d'aides pour les étudiants. L'État pourrait ainsi réaliser des économies sur plusieurs lignes budgétaires. Ainsi, en 2015 (EESRF 2017) :

1. Les aides budgétaires directes de l'État incluent les bourses et prêts pour un montant de 2038 millions d'euros, les aides au mérite pour un montant de 66 millions d'euros, les allocations de logement social (ALS) pour un montant de 1339 millions d'euros ainsi que les aides personnalisées au logement (APL) pour un montant de 242 millions d'euros. La suppression de ces aides budgétaires directes engendrerait une économie de 3686 millions d'euros.

2. Les aides budgétaires indirectes de l'État incluent, entre autres, la compensation de l'exonération des droits d'inscription dont bénéficient les étudiants boursiers pour un montant total de 107 millions d'euros.

3. Les aides fiscales incluent la majoration du quotient familial pour les étudiants rattachés au foyer fiscal de leurs parents (1 217 millions d'euros) ainsi que la réduction d'impôt pour frais de scolarité des enfants poursuivant des études dans l'enseignement supérieur ${ }^{33}$ (145 millions d'euros). La suppression des aides fiscales occasionnerait une économie de 1362 millions d'euros pour l'état. Cette suppression permettrait de surcroît de réduire le caractère anti-redistributif de ces aides à l'enseignement supérieur : en effet, si les étudiants aux revenus les plus modestes bénéficient de bourses sur critères sociaux, des APL ainsi que d'exonérations de frais d'inscription et de cotisations à la sécurité sociale, les étudiants dont les parents payent des impôts bénéficient d'avantages fiscaux d'autant plus importants que le revenu de la famille est élevée ${ }^{34}$. En dernière instance, les bourses et les APL se concentrent principalement sur les déciles de revenu les plus faibles tandis que les aides fiscales concernent essentiellement les déciles de revenu les plus élevés.

La mise en place de l'allocation universelle d'autonomie permet de supprimer ces mécanismes d'aide (soit une économie de 5,2 milliards d'euros environ) au profit d'une allocation pour tous les étudiants, quel que soit le revenu de leurs parents. Il en résulterait, pour la mise en œuvre de l'AUA, un coût net de 21,6 milliards d'euros.

\subsubsection{Financement de l'AUA}

61 La question du financement de l'AUA renvoie aux effets complexes du poids de la fiscalité sur les incitations des différents acteurs. Dans le rapport sur la stratégie nationale pour l'enseignement supérieur (STRANES), Béjean et Monthubert (2015) suggèrent trois pistes de financement: (i) porter un objectif européen de dépenses de $2 \%$ du PIB pour l'enseignement supérieur, exclu du calcul des déficits publics, (ii) dédier un troisième 
plan d'investissement d'avenir aux enjeux pédagogiques et (iii) un accroissement de la contribution des entreprises au financement de l'enseignement supérieur. Dans cet article, nous n'utilisons volontairement pas ces entrées, d'une part, parce que nous concevons l'AUA comme une nouvelle branche de la protection sociale et, d'autre part, parce que nous considérons que les choix de financement dépendront du débat national. Il en va de même pour l'accroissement des dotations récurrentes aux établissements (cf. section 3.2).

62 Le manque d'équité des aides individuelles dans l'enseignement supérieur, de même que le caractère faiblement progressif - et même nettement régressif pour les $5 \%$ les plus riches de la population (Landais et al., 2011) - du système de prélèvements obligatoires actuel (impôts et cotisations) - nous conduit à privilégier un système de financement progressifs de l'AUA, fondés sur une assiette large (pour tenir compte des fortes externalités économiques et sociales de l'éducation ${ }^{35}$ ).

Ces prélèvements pourraient également être modulés en fonction du niveau d'études de chaque contribuable, lorsque ses études ont été réalisées en France. Une telle modulation légitimerait de surcroit d'intégrer à l'assiette les revenus des individus ayant bénéficié de l'enseignement supérieur français mais non-résidents en France. Cependant, faute de bénéficier d'un accès aux données fiscales et de scolarisation permettant un chiffrage de la mesure sur cette base, nous proposons dans cet article un mécanisme de financement calqué sur celui de la branche "famille» de la sécurité sociale. Par analogie avec le système de retraite, nous proposons que des cotisations sociales alimentent une nouvelle branche (la branche « jeunesse/étudiante » - ou une rubrique de la branche « famille »).

Si le mode de financement de cette branche évolue et fait l'objet de controverses, elle reste majoritairement financée par les cotisations patronales. Ces cotisations, dont le taux est de 3,45\% pour les salaires inférieurs à 3,5 SMIC et de 5,25\% au-delà, représentent 30,2 milliards d'euros en 2016. Nous proposons de financer l'AUA par une augmentation des taux de cotisations patronales pour un montant de 21,6 milliards d'euros ${ }^{36}$.

\subsection{Accroissement des moyens des universités : chiffrage et pistes de financement}

65 Comme nous l'avons vu dans la section 3.1.2. Refonte du système d'aide et coût net de l'AUA, l'État dépense en moyenne environ 10000 euros pour un étudiant inscrit à l'université, contre 15000 pour un élève inscrit en classes préparatoires. Compte tenu du fait qu'environ 1 million d'étudiants sont inscrits en licence (RERS 2017), un alignement de la dépense par étudiant en licence sur celle en classes préparatoires (soit 5000 euros supplémentaires par étudiant et par an à l'université) induirait une dépense publique de l'ordre de 5 milliards d'euros ${ }^{37}$, finançable par l'impôt. Par cohérence du modèle d'éducation par répartition, nous supposons également une absence de frais d'inscription.

Si le financement de la mesure repose uniquement sur l'impôt sur le revenu, les 5 milliards d'euros peuvent être obtenus par un accroissement homothétique des prélèvements réalisés sur chacune des tranches de revenus, ou par une redéfinition de ses tranches afin de le rendre plus progressif. En nous appuyant sur les outils développés par Landais et al. (2011), nous avons réalisé plusieurs simulations ${ }^{38}$. L'accroissement homothétique des prélèvements obligatoires suppose, pour financer les 5 milliards d'euros, une hausse des taux marginaux d'imposition (TMI) de $7 \%$. La première tranche 
d'imposition resterait ainsi inchangée (à $0 \%$ ), la deuxième passerait de $14 \%$ à $15 \%$, la troisième de $30 \%$ à 32,1\%, la quatrième de $41 \%$ à $43,9 \%$ et la dernière de $45 \%$ à 48,2 \%. Un barème plus progressif, tel que celui présenté dans le tableau 2 constitue une autre option possible pour recouvrer les 5 milliards d'euros nécessaires. Elle permet de réduire les TMI des deux premières tranches et d'augmenter le TMI des tranches supérieures.

Tableau 2. Barème de l'impôt sur le revenu permettant de dégager 5 milliards d'euros

\begin{tabular}{|l|l|l|l|}
\hline Tranche de revenu annuel & Barème actuel & Barème homothétique & Barème plus progressif \\
\hline $0-9807$ & $0 \%$ & $0 \%$ & $0 \%$ \\
\hline $9807-27086$ & $14 \%$ & $15 \%$ & $13 \%$ \\
\hline $27086-44000$ & $30 \%$ & $32,1 \%$ & $35 \%$ \\
\hline $44000-72617$ & $30 \%$ & $32,1 \%$ & $39 \%$ \\
\hline $72617-153783$ & $41 \%$ & $43,9 \%$ & $50 \%$ \\
\hline$>153783$ & $45 \%$ & $48,2 \%$ & $60 \%$ \\
\hline
\end{tabular}

Source : Calcul des auteurs

\subsection{Quels effets pour les ménages?}

67 Dans cette section, nous étudions les effets de la mise en œuvre de l'AUA et de la mise à disposition de moyens supplémentaires pour les universités sur des foyers "types» (familles ouvrières, médianes, de cadres moyens ou de cadres du $9^{\mathrm{e}}$ décile). Pour chaque famille, nous comparons, avant et après la réforme, le revenu net du ménage (i. e. le revenu une fois déduits les prélèvements obligatoires et ajoutés les aides relatives à l'enseignement supérieur). Faute de données individuelles permettant un calcul fin, notre objectif est d'établir des ordres de grandeur. Pour ce faire, nous formulons les hypothèses simplificatrices suivantes :

1. Selon les cas de figure, nous considérons un foyer monoparental ou un couple. Nous distinguons également les cas où les enfants, lorsqu'ils sont étudiants, habitent ou non chez leurs parents. Quatre ménages «types» sont ainsi présentés ci-dessous à titre d'illustrations. Nous avons utilisé les données de l'INSEE sur la distribution des revenus en fonction de la profession pour calibrer nos familles «types » (tableau 3).

2. Nous distinguons trois périodes dans la vie de ces ménages. (i) Une période antérieure à l'entrée de(s) l'enfant(s) dans l'enseignement supérieur. Les foyers acquittent alors leur impôt sur le revenu selon l'un des barèmes proposés dans la section 3.3. Ils bénéficient toujours d'une demi-part fiscale par enfant à charge. (ii) Une période pendant laquelle un (ou plusieurs) enfant(s) est (sont) inscrit(s) dans l'enseignement supérieur. Contrairement à la situation actuelle, dans laquelle l'étudiant (ou sa famille) bénéficie de prestations sociales (bourses, ALS, APL) ou d'exonérations fiscales (réduction forfaitaire et demi part fiscale) et supporte des droits de scolarité ${ }^{39}$, ces mécanismes sont supprimés dans le cas où l'AUA est mise en place. (iii) Une période postérieure à la sortie de (ou des) l'enfant(s) de l'enseignement supérieur. Pendant cette période, nous supposons que les enfants ne sont alors plus à la charge de leurs parents. 
3. Pour chaque ménage "type » et pour chaque période, nous comparons le revenu disponible net du ménage dans le cadre actuel et dans celui d'une éducation par répartition. Nous envisageons deux cas pour le barème des taux marginaux d'imposition sur le revenu afin de simuler notre proposition d'une éducation par répartition: le cas d'un barème « homothétique » et celui d'un barème plus progressif (tableau 2). Les dernières lignes des tableaux 4 à 7 présentent une approximation de l'effet global sur le revenu du ménage à la suite de la mise en place d'une éducation par répartition. Pour cela, nous envisageons une période de 40 ans sur laquelle le niveau de revenu utilisé correspondant à un pseudo « revenu permanent ».

4. Dans l'ensemble des simulations réalisées, le cadre analytique reste inchangé: nous supposons que le premier enfant entre dans l'enseignement supérieur à 18 ans pour une durée de trois ou cinq ans, sans redoublement et sans réorientation. Dans le cas où la famille a deux enfants, nous supposons arbitrairement que ceux-ci ont trois ans d'écart. Nous pouvons d'abord distinguer deux sous-périodes au sein de la première période : la famille a d'abord un seul enfant à charge durant trois ans jusqu'à la naissance du second enfant, puis deux durant quinze ans jusqu'à l'entrée du premier enfant dans l'enseignement supérieur. Dans le cas où les deux enfants font un master, nous pouvons ensuite distinguer jusqu'à trois sous-périodes au sein de la deuxième période: la première sous-période correspond au moment où seul le premier enfant est dans l'enseignement supérieur pour une période de trois ans (le second enfant étant encore au lycée), vient ensuite une deuxième période de deux ans durant laquelle les deux enfants sont dans l'enseignement supérieur en même temps (le premier enfant fait son master durant que le second entame sa licence) et une troisième période de trois ans durant laquelle seul le second enfant est en étude.

Tableau 3. Distribution du revenu salarial annuel par catégorie socioprofessionnelle en 2015

\begin{tabular}{|l|l|l|l|l|}
\hline & Cadres & Professions intermédiaires & Employés & Ouvriers \\
\hline D1 & 11200 & 5610 & 1570 & 1950 \\
\hline Q1 & 24920 & 15910 & 5910 & 7500 \\
\hline D5 (Médiane) & 34950 & 23100 & 14240 & 16330 \\
\hline Q3 & 47990 & 28950 & 19600 & 21070 \\
\hline D9 & 68490 & 35400 & 24680 & 25620 \\
\hline
\end{tabular}

Source : INSEE (2017a) éducation par répartition sur les budgets des ménages sont présentés dans les tableaux 4 à 7 (annexes 1 à 4$)$.

Dans le premier cas - annexe $1 /$ tableau 4 -, nous supposons que le ménage est composé d'un parent ouvrier et de deux enfants. Les deux enfants n'habitent plus au domicile familial pendant leurs études supérieures. Le premier enfant atteint le niveau licence, tandis que le second atteint le niveau master. Avec trois ans d'écart, les deux enfants ne se retrouvent donc pas au même moment dans l'enseignement supérieur. Le régime d'éducation par répartition se traduit, pour ces familles, par une augmentation nette de leur revenu sur 40 ans de 8,68 \% avec les deux barèmes simulés. 

de deux parents, ayant des revenus se situant dans la médiane de la distribution des revenus en France (INSEE 2017b), et de deux enfants. Ces derniers n'habitent plus au domicile familial pendant leurs études supérieures. Nous faisons l'hypothèse qu'ils atteignent tous les deux le niveau master. Avec trois ans d'écart, il existe donc une période de deux ans pendant laquelle les deux enfants se retrouvent en même temps dans l'enseignement supérieur. Le régime d'éducation par répartition se traduit, pour ces familles, par une augmentation nette de leur revenu sur 40 ans de $5,42 \%$ avec le barème homothétique de l'impôt sur le revenu et de 6,21 \% avec le barème plus progressif. de deux parents cadres et de deux enfants. Le premier enfant fait des études jusqu'en licence et le second jusqu'en master, de telle sorte qu'ils ne se retrouvent pas en même temps dans l'enseignement supérieur. Le régime d'éducation par répartition se traduit, pour ces familles, par une augmentation de leur revenu sur 40 ans de $2,16 \%$ avec le barème homothétique de l'impôt sur le revenu et de 3,08\% avec le barème plus progressif.

(a) quatrième cas - annexe 4/tableau 7 -, nous supposons que le ménage est composé de deux parents cadres dont les revenus se situent dans le $9^{\mathrm{e}}$ décile de la distribution des revenus. Les deux enfants habitent au domicile familial pendant leurs études supérieures. Ils atteignent tous les deux le niveau master. Avec trois ans d'écart, il existe donc une période pendant laquelle les deux enfants se retrouvent en même temps dans l'enseignement supérieur. Le régime d'éducation par répartition se traduit, pour ces familles, par une diminution nette de leur revenu sur 40 ans de $0,14 \%$ avec le barème homothétique de l'impôt sur le revenu et de 1,83 \% avec le barème plus progressif.

73 Il résulte de l'analyse et de la comparaison de ces cas, comme d'autres simulations que nous avons réalisées, que, globalement, les familles aux revenus les plus modestes (familles ouvrières et médianes) voient leurs revenus nets augmenter avec la mise en place d'une éducation par répartition. Il en va de même pour les cadres se situant au niveau de la médiane de la distribution des revenus de cette catégorie. En revanche, les ménages de cadres appartenant au $9^{e}$ décile de la distribution des revenus de cette catégorie et les ménages sans enfant (ou sans enfant intégrant l'enseignement supérieur) supportent une partie du coût de ce dispositif, en complément du financement effectivement supporté par les entreprises soumises aux cotisations patronales.

\section{Conclusion}

Après avoir rappelé qu'un régime par capitalisation ne garantissait ni équité, ni efficience, ni même le financement de l'enseignement supérieur, nous avons défini ce que pourrait être un régime d'éducation par répartition et discuté de ses atouts et limites, pour construire une approche théorique du concept. Le modèle d'éducation par répartition que nous proposons conjugue la gratuité de l'accès, une allocation universelle d'autonomie et un accroissement des dépenses publiques destinées aux universités (jusqu'à un niveau jugé raisonnable pour que celles-ci puissent mener à bien leurs missions). Il est assorti d'un ensemble de conditions/mécanismes garantissant l'équité et l'efficience du système d'enseignement supérieur tout en assurant un financement pérenne et peu sensible aux conjonctures. En finançant mieux les filières dans lesquelles 
les étudiants issus de mieux modestes sont surreprésentés et en offrant un accès, identique pour tous les étudiants, à un minimum de ressources, il contribue à l'égalité des chances et réduit les risques de voir des étudiants issus de milieux modestes renoncer à leurs études, y compris prestigieuses. Ensuite, en reposant sur une fiscalité nationale et progressive (éventuellement étendue aux non-résidents ayant fait des études dans le pays), de même qu'en tenant compte à la fois des rendements privés et des rendements sociaux de l'éducation, le régime par répartition est susceptible de renforcer l'équité contributive. Pour lutter contre les risques d'anti-sélection et d'aléa moral, ce modèle repose sur l'obligation, pour tout étudiant, d'accepter l'AUA et l'engagement associé, l'absence de cumul de rémunération, le conditionnement de la prorogation de l'AUA à l'assiduité, à la réussite d'une partie des examens et au respect des conseils d'orientation, une information approfondie sur les prérequis et les débouchés des différentes filières. Notre travail a été complété par un chiffrage du régime d'éducation par répartition (en année pleine, 5 milliards d'euros pour accroître les besoins de financement des universités et 19 milliards d'euros pour l'allocation d'autonomie) et une proposition de modalités de financement (respectivement par un accroissement de $7 \%$ de chacune des tranches de l'impôt sur le revenu et par l'accroissement 3 points des cotisations de la branche famille de la sécurité sociale). Nous avons enfin estimé, à travers des simulations, l'impact du régime d'éducation par répartition sur les revenus nets de foyers types: le coût de la réforme repose sur les foyers sans enfant ou les foyers les plus aisés avec enfants.

Ce travail, qui ouvre un débat théorique et de politique économique, présente des limites qui constituent autant de pistes de recherche pour de futurs travaux. Il conviendrait ainsi de s'intéresser aux mécanismes de polarisation au sein de l'enseignement supérieur, à leurs effets et au rôle de régulation que pourrait jouer une éducation par répartition. Il conviendrait également de renforcer les estimations et simulations proposées en modélisant plus finement les revenus, les effets induits par un relèvement des prélèvements obligatoires, le choix d'assiettes plus larges ou de mécanismes limitant l'impact de la réforme sur les familles modestes sans enfant.

Enfin, il conviendrait de penser - mais cela dépasse le cadre de cet article - les politiques de logement étudiants, les modalités d'intégration des étudiants dans l'enseignement supérieur (notamment des années propédeutique) ou le lien avec l'ensemble des cycles éducatifs. Face aux résultats complexes du fonctionnement d'un marché imparfait dans le cadre d'une éducation par capitalisation, l'option d'une éducation par répartition offre donc aussi l'opportunité d'un débat transparent sur les grands enjeux éducatifs et susceptibles de donner toute leur place aux étudiants-citoyens.

\section{BIBLIOGRAPHIE}

Barr N. (1993), « Alternative funding resources for higher education », The Economic Journal, vol. 103, n 418, p. 718-728. 
Beffy M., Fougère D. \& A. Maurel (2009), « L'impact du travail salarié des étudiants sur la réussite et la poursuite des études universitaires ", Économie et Statistique, vol. 422, p. 31-50.

Behrman J. \& N. Stacey (1997), The Social Benefits of Education, University of Michigan Press.

Béjean S. \& B. Monthubert (2015), Pour une société apprenante - Propositions pour une stratégie nationale de l'enseignement supérieur (Rapport sur la STRANES), ministère de l'Éducation nationale, de l'Enseignement Supérieur et de la Recherche, septembre.

Belfield C., Britton J., L. Dearden \& L. van der Erve (2017), Higher Education Funding in England: past, present and options for the future, Institute for fiscal studies, Briefing Note 211.

Boudon R. (1974), Education, Opportunity, and Social Inequality: Changing Prospects in Western Society, New York, Wiley-Interscience.

Boudon R. (1994), The Art of Self-persuasion: the Social Explanation of False Beliefs, Cambridge, Polity Press.

Bourdieu P. (1974), « Avenir de classe et causalité du probable », Revue française de sociologie, vol. 15, p. 3-42.

Bourgeois-Pichat J. (1978), «Le financement des retraites par capitalisation », Population, vol. 33, $\mathrm{n}^{\circ}$ 6, p. 1115-1136

Breen R. \& J. Goldthorpe (1997), « Explaining educational differentials: towards a formal rational action theory », Rationality and Society, vol. 9, n 3, p. 275-303.

Cameron S. \& J. Heckman (2001), « The dynamics of educational attainment for black, hispanic, and white males ", Journal of Political Economy, vol. 109, n³, p. 455-499.

Casta A. (2015), « L'enseignement à but lucratif en France à l'aune des porosités public/privé : un état des lieux », Formation emploi n 132, p. 71-90.

CERC (2003), Éducation et redistribution, Paris, La documentation française.

Chomsky N. (2010), Réflexions sur l'Université, Paris, Raisons d'agir.

Courtioux P. (2009), « Peut-on financer l'éducation du supérieur de manière plus équitable? », Position Papers, EDHEC.

Davanne O., Lorenzi J.-H. \& F. Morin (1998), Retraites et Épargne, Rapport du Conseil d'Analyse Économique, Paris, La découverte.

EESRF (2017), L'état de l'enseignement supérieur et de la recherche en France, $\mathrm{n}^{\circ} 10$, Ministère de l'Enseignement Supérieur et de la Recherche.

Espeland W. \& M. Sauder (2007), « Rankings and reactivity: how public measures recreate social worlds ", American Journal of Sociology, vol. 113, n 1, p. 1-40.

Field E. (2009), « Educational debt burden and career choice: evidence from a financial aid experiment at NYU law school », American Economic Journal: Applied Economics, vol. 1, nº 1, p. 1-21.

Flacher D. \& Harari-Kermadec H. (2013), « Tuition fees, self-esteem and social heterogeneity », Education Economics, vol. 22, n² 2, p. 191-210.

Flacher D., Harari-Kermadec H. \& L. Moulin (2012), « Régime par répartition dans l'enseignement supérieur : fondements théoriques et estimations empiriques ", Document de travail du CEPN, $\mathrm{n}$ - 2012-06, 33 p.

Flacher D., Harari-Kermadec H. \& L. Moulin (2013), « Faut-il (vraiment) augmenter les frais d'inscription à l'université ?», Revue française d'économie, vol. 27, n³, p. 145-183. 
Friedman M. (1955), Economics and the Public Interest, Piscataway, NJ, Rutgers University Press.

Gary-Bobo R. \& A. Trannoy (2005), « Faut-il augmenter les droits d'inscription à l'université ?», Revue française d'économie, vol. 19, n 3, p. 189-237.

Giret J.-F. (2011), De l'enseignement supérieur de masse à l'économie de la connaissance : la valeur des diplômes en question, Rapport d'habilitation à diriger des recherches, Université de Bourgogne.

Glennerster H., Merrett S. \& G. Wilson (1968), « A graduate tax », Higher Education Review, vol. 1, n - 1, p. 26-38.

Glennerster H., Merrett S. \& G. Wilson (2003), « A graduate tax revisited », Higher Education Review, vol. 35, p. 25-40.

Grégoir S. (2008), « Les prêts étudiants peuvent-ils être un outil de progrès social ? ", Position Papers, EDHEC.

Grossman M. (2006), « Education and nonmarket outcomes ", Handbook of the Economics of Education, Erik Hanushek \& Finis Welch (eds), t. 1, Elsevier, p. 577-633.

Holm A. \& M. Jaeger (2008). « Does relative risk aversion explain educational inequality? A dynamic choice approach », Research in Social Stratification and Mobility, vol. 26, n 3, p. 199-219. INSEE (2017a), Le revenu salarial s'établit à 20540 euros en moyenne en 2015, INSEE Focus, $n^{\circ} 101$. INSEE (2017b), France portrait social, INSEE Références.

Jarousse J.-P. (1984), « Les contradictions de l'université de masse dix ans après (1973-1983) », Revue française de sociologie, vol. 25, $\mathrm{n}^{\circ}$ 2, p. 191-210.

Landais C., Piketty T. \& E. Saez (2011), Pour une révolution fiscale - Un impôt sur le revenu pour le XXI siècle, Paris, Le Seuil, coll. « La République des idées ».

Larsen K. \& S. Vincent-Lancrin (2002), « Le commerce international de services d'éducation : estil bon? est-il méchant? ", Politiques et gestion de l'enseignement supérieur, vol. 14, n³, p. 9-50.

Levy J. (2004), Student finance schemes in Norway: a case study, IIEP, Paris, Unesco.

Lévy-Garboua L. (1976), « Les demandes de l'étudiant ou les contradictions de l'université de masse ", Revue française de sociologie, vol. 17, $\mathrm{n}^{\circ} 1$, p. 53-80.

Lichtenberger Y. \& A. Aïdara (2011), Faire réussir nos étudiants, faire progresser la France : propositions pour un sursaut vers la société de la connaissance, Projet 2012, contribution $n^{\circ} 12$, Terra Nova.

Lipman Hearne (2010), « Wondering what works? The changing marketing mix in higher education », Key insights.

Lochner L. \& A. Monge-Naranjo (2015), « Student Loans and Repayment: Theory, Evidence and Policy ", NBER Working Paper, $\mathrm{n}^{\circ} 20849$.

Maguain D. (2005), «Les prêts contingents aux étudiants dans les pays de l'OCDE », Revue française d'économie, vol. 20, n², p. 51-71.

Maurin E. (2007), « Université : un autre financement est possible », La vie des idées, Collège de France.

Mc Mahon W. (1999), Education and Development: Measuring the Social Benefits, Oxford University Press.

Moulin L. (2014), Frais d'inscription dans l'enseignement supérieur : enjeux, limites et perspectives, Thèse de doctorat, Université Paris 13. 
Moulin L. (2015a), « Doit-on faire les frais des droits d'inscription? », La vie des idées, Collège de France.

Moulin L. (2015b), « Frais d'inscription dans l'enseignement supérieur et régimes d'Étatprovidence : une analyse comparative ", Éducation et sociétés, vol. 36, n², p. 119-141.

Moulin L., Flacher D. \& H. Harari-Kermadec (2016), « Tuition fees and social segregation: lessons from a natural experiment at the University of Paris 9-Dauphine ", Applied Economics, vol. 48, $\mathrm{n}$ - 40, p. 3861-3876.

Newfield C. (2016), The Great Mistake: How we Wrecked Public Universities and how We Can Fix Them, JHU Press.

Nicourd S. Samuel O. \& S. Vilter (2011), «Les inégalités territoriales à l'université ? Effets sur les parcours des étudiants d'origine populaire ", Revue française de pédagogie, vol. 176, n 3, p. 27-40.

OCDE (2017), Regards sur l'éducation 201 : les indicateurs de l'OCDE, Paris, OCDE Publishing.

OVE (2017a), Enquête nationale conditions de vie des étudiant-e-s 2016 - l'activité rémunérée des étudiants , Observatoire de la vie étudiante.

OVE (2017b), Enquête nationale conditions de vie des étudiant-e-s 2016 - le logement étudiant, Observatoire de la vie étudiante.

Palier B. (2003), La réforme des retraites, Paris, Puf, Coll. « Que sais-je ?», 128 p.

Psacharopoulos G. \& H. Patrinos (2004), « Returns to investment in education: a further update », Education Economics, vol. 12, n², p. 111-134.

RERS (2017), Repères et références statistiques, DEPP, Ministère de l'Éducation Nationale.

Sauviat C. (2003), « Les effets conjugués des faillites et de la baisse de la bourse sur les régimes complémentaires de retraite par capitalisation ", Chronique internationale de l'IRES n 81, p. 3-16.

Trannoy A. (2006), « Financement des universités, financement des études », Revue d'économie politique, vol. 116, $\mathrm{n}^{\circ} 6$, p. 745-782.

Van de Velde C. (2008), Devenir adulte : sociologie comparée de la jeunesse en Europe, Paris, Puf, 278 p.

Vinokur A. (2007), «Study now, pay later : partage des coûts, endettement étudiant et restructuration de l'enseignement supérieur », in A. Vinokur, Pouvoirs et financement en éducation, Paris, L'Harmattan.

Vinokur A. (2009), « La querelle des droits d'inscription », Revue du MAUSS, vol. 33, n 1, p. 441-453.

Winston G. (1999), « Subsidies, hierarchy and peers: the awkward economics of higher education ", Journal of Economic Perspectives, vol. 13, n 1, p. 13-36.

Wolfe B. \& R. Haveman (2002), « Social and nonmarket benefits from education in an advanced economy », Federal Reserve Bank of Boston Conference Series, vol. 47, p. 97-142.

\section{NOTES}

1. On pourra se référer à Bourgeois-Pichat (1978) pour une discussion des systèmes de retraite par capitalisation et par répartition.

2. Nous renvoyons à Flacher et al. (2013) pour une discussion autour de la notion d'équité.

3. Cf. Breen \& Goldthorpe (1997) ou Holm \& Jaeger (2008). 
4. Les PARC sont des prêts accordés aux étudiants pour financer leurs études et qui ne sont remboursés que si le revenu de la personne diplômée dépasse un certain seuil. C'est à Friedman (1995) que l'on doit leur conception. Leur principe repose sur l'idée que, s'il lui apparaît raisonnable de faire payer directement le bénéficiaire des études, il lui apparaît aussi essentiel de ne pas se priver des talents issus des classes populaires. Face à l'absence de contrepartie tangible à un prêt étudiant, face aux craintes des étudiants de ne pas pouvoir rentabiliser leur investissement éducatif et du fait de l'existence d'externalités positives à l'éducation, il importe donc que l'État fournisse une garantie permettant d'éviter un sous-investissement éducatif.

5. Voir par exemple Vinokur (2009).

6. "C'est ainsi que le triplement du niveau des frais d'inscription en Angleterre en 2012, qui s'est accompagné d'une baisse des subventions aux établissements de $40 \%$, risque, à la suite de l'augmentation du taux de défaut sur les prêts étudiants, de coûter plus cher au gouvernement que l'ancien système" (Moulin 2015a, p. 4).

7. Néanmoins, il convient aussi de remarquer, comme Maguain (2005), que s'ils sont souvent mis en place pour accompagner l'accroissement des frais d'inscription, les PARC peuvent également l'être dans la perspective d'un enseignement supérieur sans frais d'inscription, comme c'est le cas en Suède.

8. Il en allait de même des étudiants de l'École Polytechnique (recrutés en qualité d'officiers militaires) mais dont les obligations correspondantes (respect de l'engagement ou remboursement de la "pantoufle») ont été abandonnées en 1999 (cf. article 9 du Décret n • 70-323 du 13 avril 1970 - version consolidée du 17 septembre 2000).

9. Une modulation en fonction du coût local de la vie pourrait également être envisagée.

10. Source: EESRF (2017). Notons que depuis 2007, la LOLF (Loi Organique relative aux Lois de Finances) conduit à intégrer aux dépenses des universités celles des IUT (Instituts Universitaires de Technologie), ce qui a pour effet d'accroître artificiellement le coût moyen d'un étudiant en université.

11. Calculs relatifs à la génération de 1970 , avec 2005 pour année de référence. Cette disparité correspond vraisemblablement davantage à un manque de dotation pour les universités qu'à une surdotation des autres institutions, à l'exception, peut-être, des plus grandes écoles.

12. Il s'agit des dépenses annuelles des établissements d'enseignement par étudiant inscrit dans l'enseignement tertiaire.

13. Comme le soulignent Davanne et al. (1998, p. 15) : «[...] en première analyse, la différence entre répartition et capitalisation est indiscutable. Dans un régime en capitalisation, actifs et retraités possèdent un patrimoine pour assurer leurs besoins futurs. En répartition, ce "patrimoine" est composé de droits sur les régimes de retraite et ces droits n'ont pas pour contrepartie du capital investi dans l'économie. Ces droits sont aussi appelés "dette implicite du système de retraite" ". Dans notre approche, l'éducation par répartition se distingue également de l'éducation par capitalisation car les droits ouverts à l'AUA n'ont pas pour contrepartie un capital investi.

14. Voir Psacharopoulos et Patrinos (2004) pour une revue de littérature sur les retours liés à l'éducation.

15. Pour ce qui est des retraites, la liberté individuelle est en réalité largement encadrée institutionnellement (Sauviat 2003). C'est également le cas pour l'endettement étudiant qui dépendrait principalement du niveau des frais autorisés par l'État et des éventuelles bourses ou réduction de coûts. Du point de vue de sa logique cependant, le régime par capitalisation se présente comme se fondant sur la responsabilité individuelle.

16. Il s'agit ici à la fois de l'autonomie vis-à-vis du cercle familial, mais également des pressions associées au poids de la dette. La dette peut en effet conduire les actifs, sur lesquels elle pèse, à une relative « docilité » à l'égard de l'employeur, sur le marché du travail (Chomsky 2010). 
17. Selon l'OVE (2017a), près de la moitié des inscrits dans l'enseignement supérieur exerce une activité rémunérée pendant l'année universitaire (parmi eux, 25,1\% des étudiants ont une activité durant moins de 3 mois, 22,6\% des étudiants ont une activité rémunérée dont la durée est comprise entre 3 et 6 mois et 52,3\% des étudiants ont une activité rémunérée dont la durée est supérieure à 6 mois), qui pour 55,4\% d'entre eux n'est pas liée à leurs études. À partir des enquêtes Emploi de 1992 à 2002, Beffy et al. (2009) montrent que l'occupation d'un emploi régulier pour un étudiant réduit significativement sa probabilité de réussite à l'examen de fin d'année universitaire. Ainsi, le taux de réussite est en moyenne de $66 \%$ pour les étudiants nonsalariés contre un peu moins de $38 \%$ pour les étudiants travaillant plus de 16 heures par semaine.

18. Si, selon Barr (1993, p. 26) et Grégoir (2008, p. 721), mettre en place une telle fiscalité est équivalent à l'instauration de frais d'inscription et de PARC - dans les deux cas, il s'agit en effet de récupérer sur le revenu de l'actif ce dont il a bénéficié pour ses études - nous considérons, comme Field (2009), qu'il existe une aversion à l'endettement qui rend les deux mécanismes non interchangeables : l'impôt ou les cotisations sociales apparaissent dès lors comme préférables à l'endettement pour financer le système éducatif.

19. Il pourrait également être demandé aux non-résidents ayant bénéficié d'études en France, de contribuer au financement du système. Cette contribution pourrait s'inspirer des conventions bilatérales établies par les États-Unis dans le domaine fiscal pour leurs ressortissants installés à l'étranger dans des pays à la fiscalité plus légère. Elle pourrait aussi résulter d'un contrat entre l'étudiant et le pays qui l'a formé. Une telle perspective, qui ne manquerait pas de poser des problèmes pratiques, dépasse néanmoins le cadre de cet article. Cependant, cette règle présenterait l'avantage de réduire les problèmes d'aléa moral attaché à ce type de financement (certains étudiants préférant bénéficier de la gratuité de l'enseignement supérieur sans la financer, en s'installant hors du pays). Notons par ailleurs que le rôle accru des régions dans le financement de l'enseignement supérieur français comporte le même type de risque, les étudiants pouvant choisir de s'établir dans une autre région que celle ayant contribué au financement de leurs études, ce qui participe à la dynamique de polarisation.

20. Cette conséquence ne devrait cependant pas empêcher une régulation des pratiques des écoles concernées. Il conviendrait en effet, dans un système par répartition, de tendre vers une quasi-gratuité de l'ensemble des filières afin de ne pas induire une polarisation artificielle et inefficiente (car liée à une régulation asymétrique).

21. Ces deux mécanismes relevant également de l'aspect efficience de notre proposition, nous les développons dans la section suivante (2.4. Efficience d'un système d'éducation par répartition).

22. Les recommandations de cette commission ne doivent pas fermer la porte de certaines études mais s'efforcer, en fonction des choix de chaque étudiant, de proposer les étapes les plus pertinentes pour y parvenir.

23. Les universitaires, membres de la commission, devront être indépendants : les avis rendus ne doivent pas concerner les étudiants demandant une inscription dans leur université d'affectation, de manière à limiter les biais dans la procédure. Cela suppose notamment que les universités ne soient pas incitées à garder des étudiants dans le système pour des raisons de financement de leur activité.

24. De ce point de vue, il ne s'agit pas de répliquer le modèle des universités suédoises qui, tout en ayant l'obligation d'admettre l'ensemble des candidats, rend quasi-obligatoire l'orientation des candidats pour tenir compte des besoins sociaux (cf. Trannoy 2006, p. 770).

25. Comme le note Gregoir (2008), aux Pays-Bas, « En cas d'erreur d'orientation, un arrêt précoce dans l'année peut entrainer une annulation de la dette» (Gregoir 2008, p. 24). Dans le cas de l'AUA, un arrêt précoce dans l'année pourrait donner automatiquement droit à une nouvelle inscription avec allocation. 
26. L'application du même principe aux étudiants résidants et non résidants permet une égalité de traitement, y compris dans la participation des anciens étudiants, une fois actifs, au financement de l'enseignement supérieur. Il apparaît de surcroît que les étudiants issus de pays à bas revenus pourraient retourner dans leur pays sans avoir à supporter une charge disproportionnée, dans la mesure où les prélèvements se feraient selon les facultés contributives. Le système proposé permet donc une aide au développement et limite une fuite des cerveaux dans les pays à bas revenus dont l'origine serait liée au poids d'une dette à rembourser. Notons enfin que si le système peut profiter à des non-résidents, il est également financé, à travers la fiscalité, par des résidents n'ayant pas fait leurs études en France.

27. Notons que le principe des PARC suppose également que chaque individu rembourse en fonction de ses revenus, où qu'il ait décidé d'élire résidence. Le recouvrement n'est donc pas nécessairement plus complexe que le recouvrement d'un crédit contingent au revenu.

28. Les dépenses de marketing ont ainsi plus que doublé dans les universités américaines au cours de la décennie 2000 (Lipman Hearne 2010) ; toujours aux États-Unis, sur le long terme (1975-2011), le nombre de managers a ainsi explosé alors que celui des professeurs stagnait ( $+369 \%$ contre $+23 \%$, Newfield 2016, p. 41).

29. Comme nous l'avons souligné plus tôt dans cet article, nous entendons par polarisation le développement d'un écart entre les ressources des établissements. Cette polarisation en termes de ressources peut avoir un impact sur les écarts de qualité, la ségrégation sociale, les disparités géographiques, le développement relatif des filières ou encore les rendements des études. La polarisation peut aussi résulter de mécanismes qui ne sont pas liés, en premier lieu, aux ressources matérielles (par exemple à travers la sélection). Si le sujet de la polarisation constitue un agenda de recherche en soi, nous nous limitons ici à une approche générale essentiellement centrée sur les ressources.

30. Selon Lichtenberger et Aidara (2011, p. 14) : « la solidarité nationale garantit à l'étudiant un revenu de 800 euros par mois ".

31. Nous considérons l'ensemble des étudiants, y compris ceux inscrits dans l'enseignement supérieur privé et les apprentis. Ces deux catégories, qui connaissent un fort dynamisme (respectivement $+62 \%$ en 15 ans pour atteindre 450000 étudiants pour le privé et $+170 \%$ pour atteindre 138800 apprentis), nécessiteraient un traitement particulier : une contractualisation avec les établissements privés, non sur les programmes comme dans le secondaire mais sur les frais d'inscription (gel ou plafonnement), et un mécanisme de substituabilité ou de complémentarité entre la rémunération de l'apprenti et l'allocation universelle d'autonomie (partielle). Dans le cadre de cet article, nous ne précisons pas davantage le traitement de ces deux populations et, quitte à surestimer le coût de l'AUA, nous supposons que tout étudiant touche une allocation complète. Source : EESRF (2017).

32. Par ailleurs, il convient de garder à l'esprit que dans les pays du nord de l'Europe, là où il existe un équivalent à l'AUA, la proportion de jeunes qui n'habitent plus chez leurs parents est plus importante (Van de Velde 2008). Néanmoins, les allocations délivrées dans ces pays n'étant qu'un des éléments des systèmes institutionnels de ces pays (Moulin 2015b), nous ne pouvons en conclure que la mise en place de l'AUA inciterait les étudiants à décohabiter.

33. «Les contribuables qui ont leur domicile fiscal en France bénéficient d'une réduction de leur impôt sur le revenu lorsque les enfants qu'ils ont à leur charge poursuivent des études supérieures durant l'année scolaire en cours » (Code général des impôts, Article 199 quater F).

34. Les parents bénéficient en effet d'une demi-part fiscale (plafonnée à 1527 euros par enfant) et d'une réduction forfaitaire d'impôt (de 183 euros pour un enfant inscrit dans l'enseignement supérieur).

35. Cette proposition relève d'un large consensus entre économistes (voir notamment les travaux de Behrman \& Stacey 1997 ; Friedman 1955; Mc Mahon 1999; Wolfe \& Haveman 2002). Elle revient à considérer que les externalités positives, sur le plan économique et social, de 
l'éducation, profitent aux bénéficiaires et non bénéficiaires de l'éducation. « Une société stable et démocratique est impossible sans l'acceptation généralisée de certains ensembles de valeurs communes et sans un minimum d'alphabétisation et de connaissances de la part de la plupart des citoyens. L'éducation favorise ces deux aspects. En conséquence, le gain de l'éducation d'un enfant revient non seulement à l'enfant ou à ses parents, mais aussi à d'autres membres de la société ; l'éducation de mon enfant contribue au bien-être des autres par la promotion d'une société stable et démocratique » (Friedman 1955).

36. Faute de disposer des montants des cotisations imputables à chacun des taux, nous n'avons pu calculer l'augmentation en points que le financement de l'AUA représenterait. En utilisant les données de 2011, lorsque le taux de cotisations était unique et s'établissait à 5,4\% pour un montant total de 32 milliards d'euros, le financement de l'AUA représentait alors une augmentation de 3,1 points de cotisations (voir Flacher et al. 2012).

37. Comme indiqué précédemment, l'estimation de cet ordre de grandeur ne tient pas compte de deux effets contraires : l'accroissement probable du nombre d'étudiants susceptibles d'entrer en licence et la réduction relative du nombre d'inscrits et de réorientations (du seul fait que les étudiants bénéficieraient de meilleures conditions d'études).

38. Les simulations ont été réalisées à partir de l'outil mis en ligne par Landais et al. (2011) ; http://www.revolution-fiscale.fr/. Le simulateur repose sur la structure des revenus de 2011. Il fournit donc des ordres de grandeurs très imparfaits mais suffisants pour les besoins de l'article. 39. Les droits de scolarité utilisés dans les simulations correspondent à ceux de licence et de master en vigueur en 2017-2018 dans les universités publiques.

\section{RÉSUMÉS}

Après avoir rappelé qu'un régime par "capitalisation" ne garantissait ni l'équité, ni l'efficience, ni même le financement de l'enseignement supérieur, nous définissons ce que pourrait être un régime d'éducation par "répartition" et discutons de ses atouts et limites, pour construire une approche théorique du concept. Une éducation par répartition conjugue la gratuité de l'accès, une allocation universelle d'autonomie et un accroissement des dépenses publiques destinées à l'université. Nous montrons qu'un tel régime peut se révéler équitable et efficace pour financer le système éducatif et discutons des conditions permettant à un tel système d'être efficient. Nous chiffrons le coût du régime par répartition (en année pleine : 5 milliards d'euros pour accroître les besoins de financement des universités et 21,6 milliards d'euros pour l'allocation d'autonomie), proposons des modalités de financement et analysons l'effet de ce régime sur les revenus de foyers types. Nous concluons en présentant les limites et perspectives de recherche.

The article first highlights the limits of "self-funded education schemes" which appear to be neither equitable nor efficient, and not even effective for funding the higher education system. Then, we draw the theoretical basis of a "contributory education-funding scheme" and discuss the related pros and cons. Such a system is based on free access to education as well as an universal allowance to guarantee students' autonomy. It also includes sufficient public financing of higher education. We show that this type of scheme can lead to equitable and effective scheme for financing higher education. We also discuss the conditions for the scheme to become efficient. Finally, the article presents an evaluation of the yearly implementation cost of such a 
scheme in France (additional €5bn for financing higher education and €19bn for students' expenditure) and illustrate the effect of the scheme on some typical households.

\section{INDEX}

Keywords : universal autonomy allowance, contributory scheme, funded education scheme, financing higher education, equity, efficiency

Mots-clés : allocation universelle d'autonomie, capitalisation, répartition, financement de l'enseignement supérieur, équité, efficience

\section{AUTEURS}

\section{DAVID FLACHER}

Professeur des universités, Université de Technologie de Compiègne, COSTECH

\section{HUGO HARARI-KERMADEC}

Maître de conférences, ENS Paris Saclay

\section{LÉONARD MOULIN}

Chargé de recherche, Institut national d'études démographiques (INED), F-75020 Paris, France ; leonard.moulin@ined.fr 